\title{
Volume Rendering With Data Parallel Visualization Frameworks for Emerging High Performance Computing Architectures
}

\author{
Hendrik A. Schroots* \\ Kwan-Liu $\mathrm{Ma}^{\dagger}$
}

\begin{abstract}
Future exascale computing is demanding more and more parallelism from current software if peak computation rates are to be realized. However, exploiting this additional parallelism is not trivial. One approach is to identify finer grained parallelism using data parallel primitives (DPP). Visualization frameworks such as Dax and VTK-m are being developed using DPP for this purpose. Our work presents an exploratory study of how volume rendering maps to current and future super computing architectures. We implement a ray casting and cell projection volume renderer in Dax using DPP and compare their performance on three different hardware architectures. Despite the portability provided by these frameworks, we observe that additional architecture specific modifications are necessary to achieve acceptable performance on some architectures.
\end{abstract}

Keywords: Exascale Computing, Many Integrated Core, Data Parallel Primitives, Volume Rendering

\section{Introduction}

The standard CPU model is no longer expected to provide the necessary concurrency needed to achieve extreme scale computing. In recent years, leadership-class supercomputers have turned to using accelerators to reach petascale performance [Moreland et al. 2013]. Accelerators are highly concurrent processors such as a GPU or a many-core processor in addition to a system's primary CPU. The move from petascale to exascale computing, however, will require a disruptive change. It is estimated that a 40,000 fold increase in concurrency is needed to reach peak computational performance [Moreland 2012]. Heterogeneous computing, or systems with two or more different processor architectures that work together to achieve better performance than either one in isolation, is one possible solution.

The difficulties facing extreme scale computing are not just hardware related. With each new generation of supercomputing and hardware change, existing visualization software needs to be rewritten and adapted to reach maximum performance. Three problems facing visualization software are portability, usability and flexibility. An effort to address these issues in the context of exascale computing is under way through the research of 3 frameworks: EAVL [Meredith et al. 2012], PISTON [Lo et al. 2012], and Dax [Moreland et al. 2011]. These three frameworks are currently being combined to form a unified visualization framework called VTK-m which aims to provide the same functionality as VTK [Schroeder et al. 1996] but perform at extreme scales.

\footnotetext{
*e-mail:hschroots@ucdvais.edu

†e-mail:ma@ucdavis.edu
}

Permission to make digital or hard copies of part or all of this work for personal or classroom use is granted without fee provided that copies are not made or distributed for commercial advantage and that copies bear this notice and the full citation on the first page. Copyrights for third-party components of this work must be honored. For all other uses, contact the Owner/Author.

Copyright is held by the owner/author(s).

SA'15 Symposium on VisHPC, November $02-06,2015$, Kobe, Japan.

ACM 978-1-4503-3929-2/15/11.

http://dx.doi.org/10.1145/2818517.2818546
This paper presents two versions of a volume renderer built using the Dax framework in an effort to demonstrate the usability of it as a future visualization framework. The volume renderers span two classes of algorithms that exploit different forms of parallelism (discussed in 2.1.1 and 2.1.2). The first version is a standard volume ray caster (RC) for regular grid data. The second version attempts to exploit greater concurrency by ray casting each cell individually and globally compositing the results. We conduct a performance study of the two versions on three prevalent architectures.

The following are the main contributions of this work:

- Extend existing visualization frameworks by implementing a volume renderer for regular grid data in Dax

- Provide an in-depth analysis of the usability of data parallel primitives (DPP) for volume rendering

- A comparative study of the performance of the two volume renderers in a shared memory context across multiple architectures. The architectures are representative of what future supercomputer will likely use.

\section{Related Works}

The scope of our work draws from many different areas. We implement existing volume rendering techniques, adapt data parallel primitives to volume rendering, and use experimental visualization frameworks.

\subsection{Volume Rendering}

Volume rendering, a method for visualizing 3D discretely sampled data, can be done by image-order or object-order.

\subsubsection{Image-Order Techniques}

The most common form of volume rendering is ray casting [Levoy 1988], an image-order approach. Ray casting traces a ray from the camera's center of projection (COP) or point of view through each pixel into the scene. Each ray iterates through the volume, sampling it at regular intervals. The volume data is then mapped using a "transfer function" which determines what color and opacity a sample has. Because each ray is independent, ray casting is a highly parallel algorithm. Consequently, much work in volume rendering has demonstrated interactive performance by taking advantage of graphics hardware acceleration or multicore processors. In this study, however, our approach is hardware agnostic.

\subsubsection{Object-Order Techniques}

Object-order methods generally project each voxel or cell in storage order to achieve better efficiency [Shirley and Tuchman 1990]. The projection may be computed in a ray-casting per voxel/cell fashion. For structured-grid data, the rendering can be straightforwardly optimized, but for unstructured grid data, sorting is required. Ma and Crockett [Ma and Crockett 1997] show how to efficiently render large-scale unstructured grid volume data on distributed-memory parallel computers by carefully ordering the rendering. Child et al. 
[Childs et al. 2006] use a hybrid scheme that partitions the work for processors in the image and data domain for optimal load balancing.

\subsection{Data Parallel Primitives}

Data parallel primitives (DPP) are based on work by [Blelloch 1990]. He describes a class of algorithms that operate in $O(n \log n)$ time. These algorithms also map well to vector hardware [Chatterjee et al. 1990] and can be optimized to run exceptionally fast [Buck and Hanrahan 2003]. Algorithms such as map, reduce, gather, and scatter are the fundamental primitives used in our cell projection version. Stuart et al. [Stuart et al. 2010] employ map and reduce for volume rendering in the cloud. While we use both map and reduce in addition to other DPP, our work focuses on the performance of single node shared memory rendering.

\subsection{Visualization Frameworks}

EAVL, PISTON, and Dax are frameworks that abstract away implementation specific details [Sewell et al. 2012]. These tools allow developers to write parallel algorithms within the constructs of the framework. The framework then takes care of compiling the algorithms into a specific parallel implementation whether it be CUDA, OpenMP, Intel's Thread Building Blocks (TBB), or any future parallel programming paradigm. They are currently being combined into a single visualization framework called VTK-m, however it is still under development. Dax is aimed at extreme scale computing focusing on data parallel concurrency and has demonstrated improved performance for certain visualization algorithms such as Marching Cubes [Moreland 2014]. Both volume renderers for this work can easily be ported to VTK-m once it is complete.

Work most similar to ours is [Larsen et al. 2015], which presents a volume rendering algorithm for unstructured mesh data using only data parallel primitives in EAVL and demonstrates similar performance to existing unstructured grid rendering techniques on GPUs. Our work differs in that our implementations work with regular grid data. Regular grid data has two drawbacks compared with unstructured data. First, each vertex of a cell is transformed to find its screen space coordinates. Tetrahedral cells in unstructured grids have 4 vertices compared to the 8 for regular grid cells. Second, sampled values within a cell are trilinearly interpolated. Because of special tetrahedral properties this interpolation can be reduced to a bilinear interpolation [Shirley and Tuchman 1990] equating to faster processing per cell. The advantage of regular grids is their implicit ordering. Regular grids do not have explicit a vertex connectivity list, thus requiring less memory. Second, sorting cells to obtain correct depth order is crucial for correct rendering. Because the order is implicit, this sorting can often be avoided for regular grids.

\section{Algorithm Description}

Our first implementation is a simple ray caster (RC). By nature of the algorithm, ray casting is a map operation. For each thread of execution one for loop iterates the ray forward in space and samples the scalar field at regular intervals. As the ray is iterated it composites the color for a single pixel using the over operator eliminating the need for an explicit compositing step.

The second version exploits more concurrency by ray casting on a per-cell basis. We refer to this version as cell projection. This approach, however, exceeds the on chip memory for some processors. To illustrate, for each cell in a $200^{3}$ volume that maps to a $10 \times 10$ square of pixels, 100 color values need to be stored. Each color is a vector of 4 float values.

$$
200^{3} \text { cells } \times 100 \text { pixels/cell } \times 16 \text { bytes/pixel } \approx 11.9 \mathrm{~GB}
$$

We work with data sets much larger than $200^{3}$. To reduce the memory requirements within acceptable ranges we operate on subvolumes of the data for multiple passes.

The algorithm depends on 1 view-dependant step executed once, and 4 major steps executed for each pass. The view-dependent step identifies in which pass each cell will be processed. The four steps are Pass Identification, Screen Bounding Box, Sampling, and Compositing. We choose to divide the passes in depth order making it easier to composite results between passes. We present the algorithm in 1 .

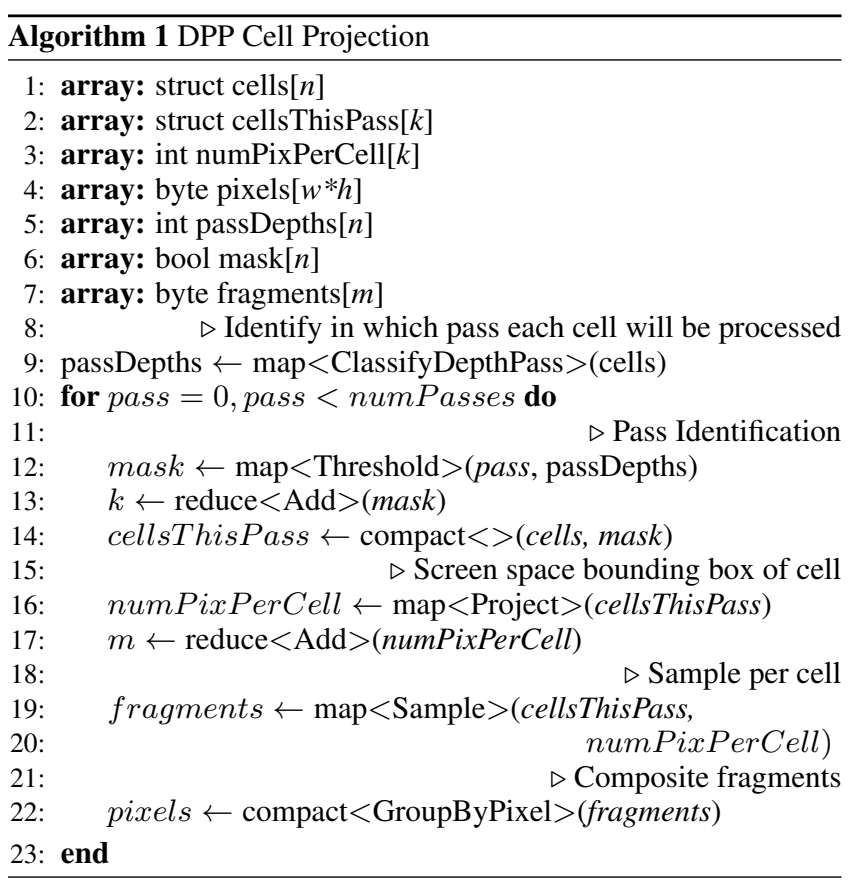

\section{Experiment Setup}

We investigate the performance of both implementations via two aspects. First, we simply compare the run times of the applications across three architectures. Second, we profile the behavior on the GPU using multiple metrics.

\subsection{Run Time Performance}

We tested both versions on three different architectures: NVIDIA Titan, Intel Xeon, and Intel Xeon Phi. On each architecture we tested a small and large data set. The small is a $216^{3}$ supernova volume which is about 10 million cells, and the large data another supernova simulations measuring $432^{3}$ voxels (about 80 million cells). All tests are rendered to a 1024 x 1024 image. 2 versions across 3 architectures for 2 data sets gives us a total of 12 test cases. While different transfer functions did affect the runtime, the difference was negligible so we chose one that best represents the interesting features of the volume.

Two techniques are considered good practice that generally improve the performance of RC: early ray termination and empty space skipping. Early ray termination was implemented in both versions, however, empty space skipping was not. Empty space skip- 


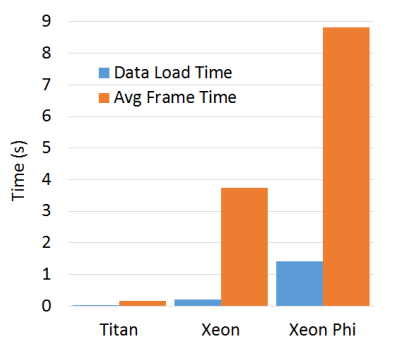

(a) Run times for RC of 10 million cells.

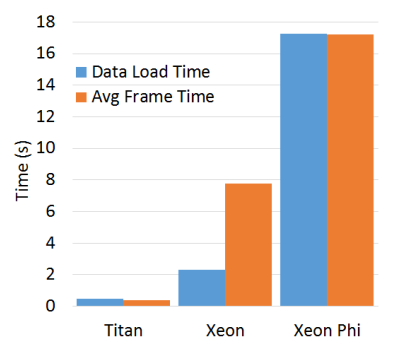

(b) Run times for RC of 80 million cells.

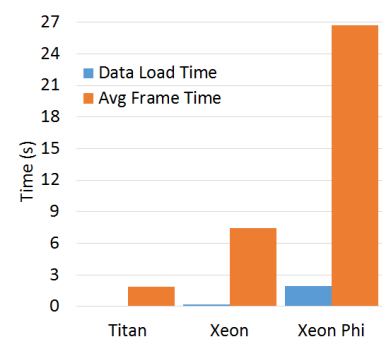

(c) Run times for cell projection of 10 million cells.

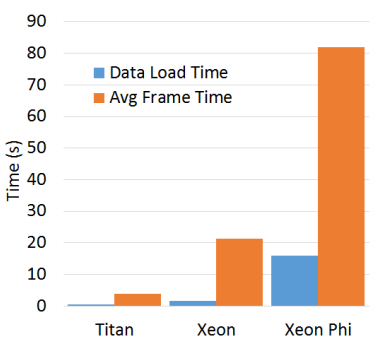

(d) Run times for cell projection of 80 million cells.

Figure 1: (a),(b) Run times of ray caster for small and large volumes respectively. (c),(d) Run times of cell projection for small (single pass) and large (4 passes) volumes respectively.

ping requires a secondary tree data structure that contains the minimum and maximum extents of contiguous regions of space within the volume that are empty for the given transfer function. Since we ran up against the memory limitation of both the Titan and the Xeon Phi the design decision to not use the additional data structure was made.

As mentioned in Section 3, the cell projection version was rendered over multiple passes. The time to render each frame grew with the number of passes which is attributed to synchronization overhead. Performance numbers reflect the smallest number of passes that still fit in memory. The small data set fit entirely into memory in a single pass while the large data set has to be rendered in 4 passes to fit into 6 GB of memory.

\subsection{GPU Profiling}

We profiled the two versions using NVIDIA's Visual Profile. While there are many attributes of an application that characterize its behavior, we chose to look at registers per thread, executed instructions per cycle (IPC), stalls due to data load requests, and occupancy. These metrics are indications of how well the hardware is being utilized and the impact of memory on access on the performance.

\subsection{Hardware Configurations}

We present the relevant performance specifications of each architecture here.

- GPU: NVIDIA Titan X, 2,688 stream processors, 6GB on chip memory, $837 \mathrm{MHz}$

- CPU: 2x Intel Xeon E5-2620, 6 cores per processor and 2x hyperthreading per core, 64GB system memory, $2.2 \mathrm{Ghz}$

- MIC: Intel Xeon Phi 5110p many integrated cores (MIC) coprocessor, 60 cores with $4 \mathrm{x}$ hyperthreading per core, $8 \mathrm{~GB}$ on chip memory, $1.1 \mathrm{GHz}$

\section{Results}

Our results show that the performance of different architectures is counter intuitive. Despite having similar peak theoretical performance the NVIDIA Titan runs order of magnitude faster than the Intel Xeon Phi. Volume data is read from an SSD. If the data is not in float format then the values are first converted to float and then normalized. We refer to this conversion and normalization as "data load time".

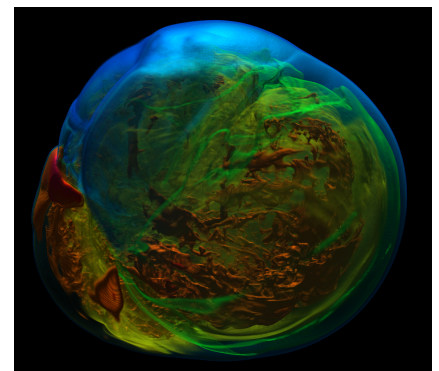

Figure 2: Rendered image of large supernova volume using the cell projection volume renderer.

\subsection{Ray Casting}

Figure 1a shows the data load time and runtime of the RC version for the small dataset. We see the Titan runs sub-second, generating about $3 \mathrm{fps}$. The Intel Xeon has data load times near that of Titan's, but renders much slower. This is attributed to the throughput difference between the two processors. We see a significant performance drop for the Xeon Phi in both data load times and frame rates.

Figure $1 \mathrm{~b}$ shows a rise in the data load times for the larger dataset. However, we can see that for the Xeon phi the data load processes grew significantly compared to the additional complexity of rendering the additional cells.

\subsection{Cell Projection}

Simply exploiting more concurrency does not always translate into better performance. The cell projection approach has concurrent threads on the order of 10 million for the small data set. We see in Figure 1c that the additional concurrency slows down the performance compared to RC in Figure 1a - 1b.

The additional concurrency grows almost another order of magnitude for 80 million cells. Figure 1d demonstrates the drop in performance despite the available concurrency. We attribute this degradation to two factors. First, increased concurrency comes with overhead, particularly memory overhead. Cell projection can be a very irregular algorithm when it comes to memory access. Second, by nature of a multiple DPP algorithm, there are synchronization points between DPP invocations. The RC version does not have this problem since the entire algorithm is a single map primitive. In addition, there are multiple passes that require synchronization between iterations. 


\subsection{GPU Metrics}

Intuitively the more registers a primitive uses the fewer the instances that can be scheduled in a single Cuda core. We expect the occupancy to drop as the register number grows. In a similar relationship the more data load stalls there are the fewer the number of instructions that are executed per cycle, so we expect the IPC to drop.

Table 1 compares the single $\mathrm{RC}$ primitive, and the individual primitives used in the cell projection version. We see that the $\mathrm{RC}$ version has a high IPC which explains why it performs to well. Additionally, we see that it is memory bound as it spends a third of the time waiting for data to be loaded.

The cell projection primitives demonstrate varied behavior. The last three primitives have high register counts so it makes sense to see low occupancy. Despite having a similar number of registers and occupancy to Bounding Box, the Compositing step has a much higher memory demand which lowers the IPC. This primitive is another memory bound bottle neck.

Table 1: Registers per thread, IPC, stalls due to data request, and occupancy compared between the RC map primitive, and the multiple primitives used for cell projection using the large data set.

\begin{tabular}{lcccc} 
Primitive & Registers & IPC & $\begin{array}{c}\text { Data } \\
\text { Load } \\
\text { Stalls }\end{array}$ & $\begin{array}{c}\text { Occu- } \\
\text { pancy }\end{array}$ \\
\hline RC (map) & 96 & 1.990 & $33.9 \%$ & $28.5 \%$ \\
\hline Pass Identify & 32 & 0.689 & $88.7 \%$ & $97.9 \%$ \\
Bounding Box & 116 & 3.227 & $6.0 \%$ & $25.0 \%$ \\
Sampling & 215 & 1.055 & $13.5 \%$ & $12.4 \%$ \\
Compositing & 110 & 0.415 & $81.5 \%$ & $22.7 \%$
\end{tabular}

\section{Conclusions}

We present a data parallel volume rendering algorithm for regular grid data written in Dax. We compare the performance a volume ray caster, also implemented in Dax, on three architectures. We demonstrate that there are trade-offs between portability and performance. Visualization frameworks like Dax and VTK-m are great at abstracting away implementation specific details making it simpler for the programmer to develop within the constructs of the framework and remain portable across multiple architectures. However, the flexibility of the frameworks sometimes comes at the cost of performance for algorithms such as RC and cell projection.

Additionally, we profile the two implementations on the GPU. We see that many of the primitives are memory bound. This leads us to conclude that a better data model, as well as more fine tuning could exploit additional parallelism and move the algorithms towards being compute bound. Future work will profile the algorithms on the Intel Xeon Phi to better understand what the bottle necks are for that architecture.

\section{Aknowledgements}

This research is sponsored in part by the U.S. Department of Energy through grants DE-SC0005373, DE-FC02-12ER26072, and DE-SC0012610. This study was run in part on the resources of the National Energy Research Scientific Computing Center.

\section{References}

BLELLOCH, G. E. 1990. Vector models for data-parallel computing, vol. 356. MIT Press.

BUCK, I., AND HANRAHAN, P. 2003. Data parallel computation on graphics hardware. unpublished report, Jan.

Chatterjee, S., Blelloch, G. E., And Zagha, M. 1990. Scan primitives for vector computers. In Proceedings SC '90, 666-675.

Childs, H., Duchaineau, M. A., And Ma, K.-L. 2006. A scalable, hybrid scheme for volume rendering massive data sets. In $E G P G V, 153-161$.

Larsen, M., Labasan, S., Navrtil, P., Meredith, J., And CHILDS, H. 2015. Volume Rendering Via Data-Parallel Primitives. In Proceedings of EGPGV'15, 53-62.

LEvoy, M. 1988. Display of surfaces from volume data. Computer Graphics and Applications, IEEE 8, 3, 29-37.

Lo, L.-T., Sewell, C., And Ahrens, J. P. 2012. Piston: A portable cross-platform framework for data-parallel visualization operators. In Proceedings of EGPGV'12, 11-20.

MA, K.-L., AND CROCKETT, T. W. 1997. A scalable parallel cellprojection volume rendering algorithm for three-dimensional unstructured data. In Proceedings of PRS '97, 95-104.

Meredith, J. S., Ahern, S., Pugmire, D., And Sisneros, R. 2012. Eavl: the extreme-scale analysis and visualization library.

Moreland, K., Ayachit, U., Geveci, B., and Ma, K.-L. 2011. Dax toolkit: A proposed framework for data analysis and visualization at extreme scale. 97-104.

Moreland, K., Geveci, B., Ma, K.-L., And Maynard, R. 2013. A classification of scientific visualization algorithms for massive threading. In Proceedings of the 8th Workshop on $\mathrm{Ul}$ trascale Visualization, 2.

MoREland, K. 2012. Oh, $\$ \backslash \#$ ! exascale! the effect of emerging architectures on scientific discovery. In High Performance Computing, Networking, Storage and Analysis (SCC), 2012 SC Companion:, IEEE, 224-231.

Moreland, K. 2014. A pervasive parallel framework for visualization: Final report for fwp 10-014707. Tech. rep., Sandia National Labs.

Schroeder, W. J., Martin, K. M., and Lorensen, W. E. 1996. The design and implementation of an object-oriented toolkit for 3d graphics and visualization. In Proceedings of $\mathrm{Vi}$ sualization '96 Conference, 93-ff.

Sewell, C., Meredith, J., Moreland, K., Peterka, T., DeMarle, D., Lo, L.-T., Ahrens, J., Maynard, R., AND GEVECI, B. 2012. The SDAV software frameworks for visualization and analysis on next-generation multi-core and manycore architectures. In In Proceedings of the 7th Ultrascale Visualization Workshop, SC '12 Companion, 206-214.

Shirley, P., AND TuChman, A. 1990. A polygon approximation to direct scalar volume rendering. In Proceedings of 1990 Workshop on Volume Visualization, 63-70.

Stuart, J. A., Chen, C.-K., MA, K.-L., And Owens, J. D. 2010. Multi-gpu volume rendering using mapreduce. In Proceedings of the 19th ACM International Symposium on High Performance Distributed Computing, ACM, New York, NY, USA, HPDC '10, 841-848. 